\title{
correspondence
}

\section{Negative friction coefficients}

To the editor - Deng et al. recently reported ${ }^{1}$ that the friction force between the tip of an atomic force microscope and a graphite surface increases with decreasing load. This phenomenon which is described as a curve with a negative slope when the friction force is plotted against load, and thus leads to a so-called negative friction coefficient - was explained by a model where the adhesion between the atomic force microscope tip and the graphite surface is stronger than the dispersion forces holding the layers in graphite together ${ }^{1}$. Here, by way of two examples of the friction between polymercoated surfaces in aqueous solution, I illustrate that such a situation is not limited to graphite or similarly structured materials, yet is a more general phenomenon related to hysteresis in the adhesive interaction between two sliding bodies.

The first example refers to the friction between layers of methylcellulose ${ }^{2}$ anchored by hydrophobic interactions to a hydrophobized silica surface and a hydrophobized silica microparticle. Figure 1a shows the corresponding friction force-versus-load curve. As a load is applied, the friction force increases linearly with the load, with a change in the friction coefficient at approximately $5 \mathrm{nN}$. It is likely that the change in the frictional response results from an irreversible reorganization of the polymer layer arising from the combination of high load and shear stress. As unloading starts at about $8 \mathrm{nN}$, the friction force continues to increase as load decreases. This may occur because the adhesion between the polymer films is stronger than the force anchoring the polymers to the substrate, thus allowing the polymer layer to be lifted as the load decreases. This explanation is similar to the one given by Deng et al. for the case of graphite ${ }^{1}$, although the two systems are fundamentally different (further details are given in the Supplementary Information).
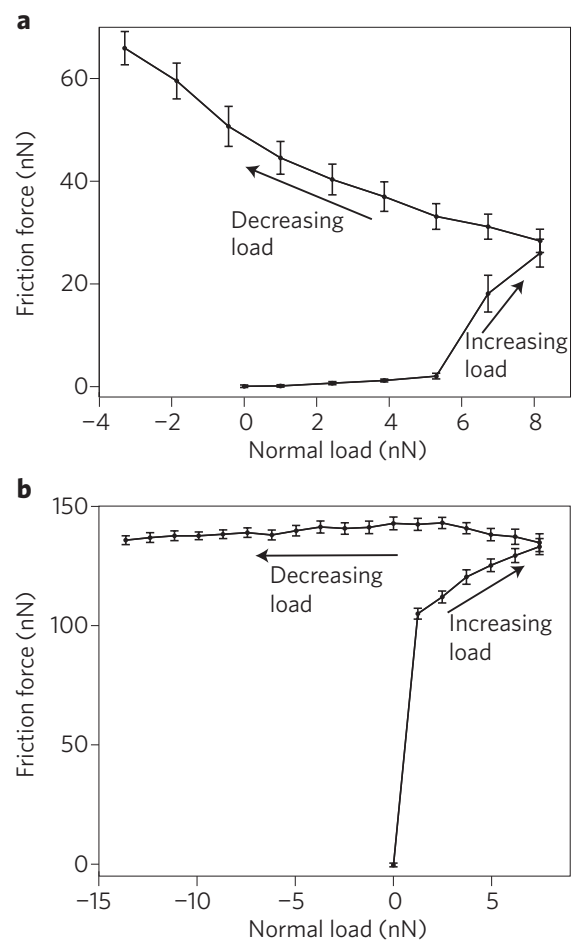

Figure 1 | Hysteresis in the friction force between two sliding bodies. a,b, Graphs showing loading-unloading cycles of the friction force between surfaces coated with methylcellulose (a) and the diblock copolymer poly( $\mathrm{N}$-isopropylacrylamide) ${ }_{48}$-block-poly(3acrylamidopropyl)trimethylammonium chloride) ${ }_{20}$ (b). Negative friction coefficients arise when the friction force increases with decreasing load. Error bars indicate standard deviations. Details about the preparation of the surfaces and on the friction-force measurements are provided in the Supplementary Information.
The second example involves the interaction between silica surfaces coated with a hydrophobic, cationic diblock copolymer ${ }^{3,4}$ by means of electrostatic anchoring. Figure $1 \mathrm{~b}$ shows that, after an initial jump to high friction resulting from adhesive contact, an almost linear increase in the friction force develops with increasing load. As the load is reduced, however, the friction force first increases and then levels off until the surfaces pull free from each other. In this case, a negative friction coefficient appears because the intermolecular interactions between the hydrophobic blocks are stronger than the forces between the cationic blocks and the silica surface. In fact, as unloading continues, the bridging polymers start to snap off, and the friction force declines. This process is almost reversible, both within loadingunloading cycles and between them.

A negative friction coefficient is therefore a special case of the common phenomenon of hysteresis in the adhesive interaction between two surfaces during a loading-unloading cycle ${ }^{4,5}$.

References

1. Deng, Z., Smolyanitsky, A., Qunyang, L., Feng, X-Q. \& Cannara, R. J. Nature Mater. 11, 1032-1037 (2012).

2. Bodvik, R., Thormann, E., Karlson, L. \& Claesson, P. M. Phys. Chem. Chem. Phys. 13, 4260-4268 (2011).

3. Loan, T. T. et al. J. Colloid Interface Sci.

342, 142-146 (2010)

4. Dedinaite, A. et al. Soft Matter 6, 2489-2498 (2010).

5. Pettersson, T., Naderi, A., Makuska, R. \& Claesson, P. M. Langmuir 24, 3336-3347 (2008).

Additional information

Supplementary information is available in the online version of the paper.

Esben Thormann

KTH Royal Institute of Technology, Department of Chemistry, Drottning Kristinas väg 51, SE-100 44 Stockholm, Sweden.

e-mail: esben@kth.se 